\title{
Moire analogies as an educational tool
}

\section{Nils Abramson}

Nils H. Abramson, "Moire analogies as an educational tool," Proc. SPIE 2525, 1995 International Conference on Education in Optics, (13 October 1995); doi: $10.1117 / 12.224068$

Event: SPIE's 1995 International Symposium on Optical Science, SPIE. Engineering, and Instrumentation, 1995, San Diego, CA, United States 
Moire analogies as an educational tool

Nils H. Abramson

Royal Institute of Technology, Materials Processing, Industrial Metrology

10044 Stockholm, Sweden

\begin{abstract}
There are many analogies between Interference patterns and Moiré patterns. Let a laser beam be represented by a transparent tape covered with a set of black stripes, that are perpendicular to the length of the tape. If the stripes are straight, parallel and have a constant separation, the tape represents a light beam that is both spatially and temporally coherent. If two such tapes intersect, moiré fringes are formed that bisect the angle between them. These fringes remain stationary even if the two tapes move in a direction parallel to their length. This is true for any velocity, as long as it is the same for the two tapes. The situation is the same for interference fringes, they bisect the angle between the two beams and the separation of the two types of fringes also obey the same equations.The interference fringes are stationary, even in spite of the fact that the two beams both move with the impressive velocity of $300000 \mathrm{~km} / \mathrm{sec}$. If you move one of the tapes seven stripes more than the other, then seven moiré fringes will pass a stationary point. It is the same with interferometry, if you move one mirror seven half waves then seven interference fringes will pass a stationary point. That is the reason why we can measure velocity by use of the Doppler shift in spite of the fact that light has the impressive frequency of more than $100000000 \mathrm{Mc}$.
\end{abstract}

Keywords: Interferometry, moire patterns, visualization, overhead projection, coherence, Doppler effect, phase stepping, heterodyning, super luminal velocities.

\title{
1. OPERATING INSTRUCTIONS
}

All explanations are analogies! New phenomena that we want to explain are compared to something already known. There is no doubt that Mathematics is a very useful analogy because it is so general. But just because of its generality, it might sometimes become very complicated. Sometimes it becomes more complicated than the phenomena it is used to explain. In that case it is better to use other analogies as we do in this book.

The amount of scientific knowledge nowadays is so large that it is impossible to memorize it all. Therefore, we must use simple analogies that span over as large an area as possible to get an inuitive feeling for how light behaves in different situations. Our method of visualizing the behavour of light is mainly based on diagrams and the analogy between interferometry and the moiré effect. Moiré patterns are formed when two sets of patterns are placed on top of each other. As an example I take two identical sets of striped pattern that represent two laser beams ( $a$ and $b$ of Fig. 1). When they are placed so that they cross each other, a moire pattern is formed representing the interference fringes caused by the two intersecting beams.

Where the two laser beams are out of phase they might cancel each other so that the result is that together they produce no light at all (destructive interference). The reason to this phenomenon is the constant phase relationship of the laser beams as. If the two beams are in phase as they hit the wall the voltage +1 added to +1 produces +2 , and the voltage -1 added to -1 produces -2 and thus the intensity of the illuminated spot increases (contructive interference). However, if the two beams are out of phase, the result is that +1 is addded to -1 and -1 to +1 so that the result is zero and the light disappears. 
In this paper, we use the simplification that the laser light wave is not a smooth curve as in Fig. 2a but is instead a square wave as in Fig. $2 b$.Further on, we let the positive phase be represented by brightness and the negative phase by darkness (Fig. 2c). Finally, we produce the striped pattern that is used for the moire effect of Fig. 1. With this analogy we see that two in-phase beams produce brightness while two out-of-phase produce darkness. This is the analogy we are going to use throughout this paper.

When two laser beams intersect, they produce interference fringes that with our method are represented by placing two transparencies of striped patterns at an angle on top of each other as was described in Fig. 1. The moiré fringes thus formed are in many respects analogous to the interference fringes. This statement is important to keep in mind for the rest of this book.

The reader of this paper should have access to a copying machine to make transparent copies of the patterns that can be used to produce the moire patterns that represent the interference fringes.

Thus, Fig. 1 shows both the resulting moire pattern and the single pattern that should be copied twice so that a live moire pattern can be formed by the reader.

The fringes of the first single pattern representing the light waves are named primary fringes. The moire pattern of the primary fringes represents interference fringes and are named secondary fringes. The moiré of pattern of the secondary fringes, e.g. holographic interference fringes are named fringes of the third order etc. The fringe pattern of a higher order always represents the difference between fringe patterns of a lower order.

There are, of course, also important non similarities between moiré fringes and interference fringes. As you add moiré fringes, the brightness never goes up. It can either be constant (representing constructive interference) or go down (representing destructive interference). Thus, the analogy between moire fringes and interference fringes is not relevant to their intensity, but only to the position and the shape of the fringes. In connection with this statement it is instructive to discuss the addition of intensities of laser beams.

When two coherent laser beams of the same intensity intersect at an angle on a screen surface, both dark and bright fringes are formed. Let us assume that half of the surface is covered by the dark fringes. Then we understand that, in order to conserve the light energy, the rest of the surface must be covered by light that has an intensity which is four times as high as that for each beam alone. Thus, we understand that intensities of coherent light do not add in a simple way. The reason is that it is not the intensities but the amplitudes that add.

The light intensity is a measure of a laser beams energy per second and square $\mathrm{cm}$. However, the intensity is a function of the square of the amplitude, very much the same way as the energy of a moving mass is a function of the square of its velocity. If, therefore, two coherent laser beams that are in phase with each other illuminate the same point, the resulting intensity is found by calculating the square of the sum of the square root of each beam. Thus:

$$
\mathrm{i}_{\mathrm{tot}}=\left(\sqrt{\mathrm{i}_{1}}+\sqrt{\mathrm{i}_{2}}\right)^{2}
$$

Where:

$i_{\text {tot }}=$ total intensity

$i_{1}=$ intensity of one beam

$i_{2}=$ intensity of other beam

Let both $i_{1}$ and $i_{2}$ have the value of unity. Then $i_{\text {tot }}$ will be 4 just as in our earlier estimate. What happens if $i_{1}$ is $1 \mathrm{i}$ and $i_{2}$ is $100 \mathrm{i}$ ? Well, $i_{\text {tot }}$ becomes $(1+10)^{2}=121 \mathrm{i}$. Thus, the coherent addition of $1 \mathrm{i}$ results in an increase in intensity of no less than 21i. Had the to beams been incoherent the resulting increase in intensity would be simply $1 \mathrm{i}$. 
Until now we have looked at constructive interference. What about destructive interference? Well, we just change the plus sign into a minus sign. Thus, for two beams of intensity $1 \mathrm{i}$, the total intensity will be zero, and for the two beams with intensities of $1 \mathrm{i}$ and $100 \mathrm{i}$ the total intensity will be $81 \mathrm{i}$. From these results we understand that if one signal beam of the intensity $1 \mathrm{i}$ varies in phase from zero to 180 degrees the output signal will vary by $40 \mathrm{i}$, if a coherent continuous beam beam of $100 \mathrm{i}$ were added.Not a bad amplification!! In this book, we will find many examples when this strange phenomenon is referred to e.g. the influence of the reference beam in holography and the advantage of heterodyning in interfereometry. However, as we have already mentioned, when the moire analogy to interferometry is used, we do not pretend to represent the intensities of the fringes but only their shapes.

\section{Coherence length}

Ideal laser light differs from ordinary light in that it consists of well ordered sinusoidal electromagnetic waves of constant wavelength that all are in phase with one another. Such a laser beam is said to be coherent. An ordinary He-Ne-laser, however, is not that good. Usually it emits light of not just one single frequency but of four or five different frequencies, and therefore different wavelengths. Along the beam these waves of different lengths will continuously work themselves alternatively in and out of phase so that the phase relationship will appear constant only over a limited length of the laserbeam. This length is named the coherence length. For a perfect laser, the coherence length can be hundreds of meters but for ordinary He-Nelasers it is usually around $15 \mathrm{~cm}$.

Let us study the experiment of Fig. 3. A laser at the left emits a beam, half of which is reflected downwards by a beamsplitter, the other half passes through and is later also reflected downwards by an ordinary mirror. Where the two beams intersect, we see interference fringes in the form of dark lines on a screen where the two laser spots meet. The interference fringes disappear if the distance between beam splitter and the mirror is longer than the coherence length. Thus, the longitudinal, or temporal, coherence length could be defined as the longest path length difference that will allow fringes to form when two light rays from the same source are split and later united again.

If two laser beams intersect, bright and dark lines, interference fringes, are produced. This phenomenon is perhaps best explained by the analogy of Fig. 1. Two tapes, covered with stripes, cross each other and dark lines, moire' fringes are formed. These moire' fringes behave analogous to the interference fringes formed if the tapes were laser beams and the stripes were individual light waves. The fringes are parallel and bisect the angle between the two tapes (beams). The larger the angle between the two tapes (beams), the more closely spaced are the fringes. If the angle is zero the whole intersection could become totally dark or totally bright (destructive or constructive interference respectively). The fringes are stationary even if the two tapes (lightwaves) move in the direction of the arrows. The speed could be zero, or it could be the speed of light, but the result would be the same. That is the reason why we can let the moire of the stationary striped tapes represent the interference of light waves. These statements are true, however, only if the two tapes move with exactly the same speed and if the separation of the stripes (the wavelength) is constant to a very high degree, analogous to laser beams of long coherence.

Summing up, the analogy between the moire fringes of and the interference fringes formed by two intersecting beams travelling from $A$ to $D$ and from $B$ to $C$ respectively of Fig. 1 may be described as follows:

\section{$\underline{\text { Moiré }}$}

White plus black stripe is $\lambda$.

Fringes bisect the angle $2 \alpha$.

\section{Interference}

Wavelength is $\lambda$.

Fringes bisect the angle $2 \alpha$. 
The straightness of the fringes depends on the straightness and equal spacing of the stripes.

If the two striped bands are moving with identical velocities from $A$ to $D$ and from $B$ to $C$, the moire patterns stay fixed.

If the striped band $A D$ is moved towards D one more stripe than $\mathrm{BC}$ is moved towards $C$, then the fringes will move downwards one fringe spacing.

The fringe spacing is $0,5 \lambda / \sin \alpha$.

When $2 \alpha$ is $0^{\circ}$ the bands will either be unifurmly striped ur totally black.

When $2 \alpha$ is $180^{\circ}$ the bands will produce a beating effect when moving with identical but opposite velocity.
The straightness of the fringes depends on the straightness and constant spacing of the wavefronts.

As the wavefronts move with the speed of light (c), the fringes stay fixed

If the phase of the beam AD is advanced one wavelength compared to $B C$, then the fringes will move downwards one fringe spacing.

The fringe spacing is $0.5 \lambda / \sin \alpha$.

When $2 \alpha$ is $0^{\circ}$ the two beams will add uniformly either constructively or destructively.

When $2 \alpha$ is $180^{\circ}$ there will be standing waves, the spacing between them being $0.5 \lambda$.

Now we have looked at the analogy between the moire pattern and the interference effect of two intersecting, mutually coherent beams of parallel light. Later we will examine the analogy between the moire pattern and the interference patterns formed by the divergent light from two close point sources of mutually coherent light. In that case, the wavefronts will be spherical and the interference fringes will consist of surfaces in space that are neither flat nor parallel. The new concept of "beams of coherent ohservation" will also be studied later to see how this can be used to calculate the shape of these three-dimensional interference surfaces. This will show how interference fringes are formed when they intersect objects.

Let us now study Fig. 4. where one tape is produced of cut pieces of different stripe separations that are about one centimeter long and glued together in a more or less random way. This tape then represents light of one centimeter coherence length and when two copies are put together at an angle we can clearly see that the interference fringes are no more aligned along straight lines that bisect the angle between the beams. Thus, if we move the tapes in the direction of the arrows, the fringes will most probably jump up and down and this movement of the interference fringes will happen with velocities in the order of the speed of light and therefore we will perceive no fringes at all. Not even if we carefully position the two copies so that each section is combined with itself will it produce uniform fringes.

The steps in height of the moire' fringes represent phase differences of the different parts along the beam. These are caused by a lack of temporal coherence. The difference in separation and direction of the moire' fringes represents a lack of spatial coherence. It is caused by small differences in the directions of the stripes (wave fronts) and therefore can be eliminated by letting all the light of each laser beam pass through a small hole (a spatial filter), at a long distance from the studied intersection. This situation represents a laser beam with a coherence length of one individual cut piece, and the jumps of the separation of the stripes represents random phases of each coherence length, which is often named wavetrain.

Now let us repeat the experiment, but this time all the cut pieces have the same stripe separation( Fig. 5). In this situation all the light is of the same wavelength. Let us also make all the stripes parallel. This 
represents the light that has passed through a small hole far away. In this case we can produce nice and uniform fringes. However, this is the case only if the two copies are positioned so that each section is combined with itself. As soon as I slightly move one copy in relation to the other then the moire' fringes are broken and no interference fringes will be seen by the eye as the beams pass by with the speed of light. Neither is it possible to produce any stationary destructive interference. The reason for this behaviour is that when I glued the cut pieces together I moved them in a random way so that the sparation of the stripes makes a jump when going from one piece to the next.

There is, however, one more special strange behaviour of the coherence that is found when laserlight is studied. The laser consists of an amplifying medium in between two parallel mirrors one of which is usually slightly transparent to let a fraction of the laser beam out. Most of the light inside the laser is reflected back and forth and each time a certain part (cut section) reaches the outgoing mirror some of it leaves the laser. The remaining part (of the same cut section) is reflected twice and then it again reaches the outgoing mirror and some of the same cut section again leaves the laser. In this way the same sections leave the laser again and again separated by double the distance between the mirrors (the laser cavity).

Thus, we can say that interference fringes are formed in our experiment if the distance between beam splitter and mirror is less than the coherence length plus zero or a number double the cavity length of the laser.

Another way to explain the same thing is seen in Fig. 6 where a light wave has a certain phase at $Q$. The light travels then to mirror $M_{1}$ and is reflected to $M_{2}$ and then again reaches $Q$. In order to produce constructive interference, this light must have the same phase as it originally had at $Q$, otherwise destructive interference is produced and the light disappeares. Thus, there must be an integral number of light waves within twice the laser length. Let us assume that the number of waves is one million. But maybe the wavelength is so flexible that it could be slightly compressed so that also one million and one waves can be pressed within the doubled laser length. Thus, we have a laser beam with two wavelengths that are in phase with each other for every double laserlength and out of phase in between. The laser therefore appears to be switching on and off with such a high frequency that it produces light pulses with a length of about twice the laser length.

In Fig. 7, we have tried to demonstrate this situation with another moire analogy. First we made one tape with uniform stripes. Then we copied this tape and at the same time demagnified the image so that, even if the number of stripes were identical, it became three stripes shorter than the original. When we placed the two tapes on top of each other, a moiré pattern was formed that had three periods within the length of the tapes. This moire pattern, which is caused by the small difference in stripe separation between the tapes, represents the beat frequency caused by the difference in wavelength between the two emitted laser frequencies. The number of moire periods represents the number of axial modes of the laser.

If you move the band $B C$ of Fig. $1 b$ to the right or to the left the fringes move upwards or downwards respectively. If you move the pattern of Fig. $7 \mathrm{~b}$ to the right or to the left the fringes of Fig. $7 \mathrm{c}$ move to the right or the left respectively. These two phenomena in combination with phase stepping or heterodyning are used to find the direction of motion in interferometers of different types.

Methods similar to those described here can be used to visualize a great number of optical phenomena such as conventional interferometry, reflection, diffraction, holographic interferometry, Doppler effects, apparent super luminal velocities in space and relativistic optical effects as will be described in reference 1.

\section{$\underline{\text { Reference }}$}

1. Nils H. Abramson, "Light -i n - flight, or, The Holo-diagram: The Columbi egg of Optics", book to be published by SPIE 


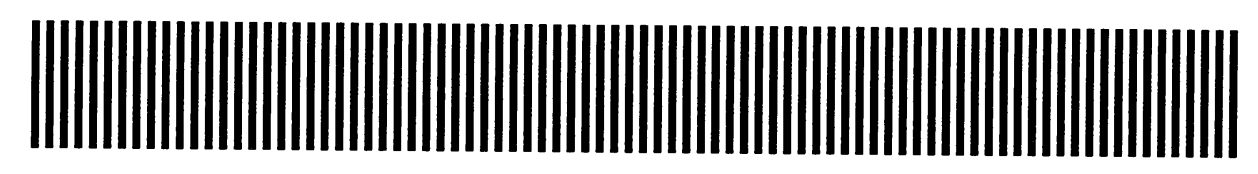

a

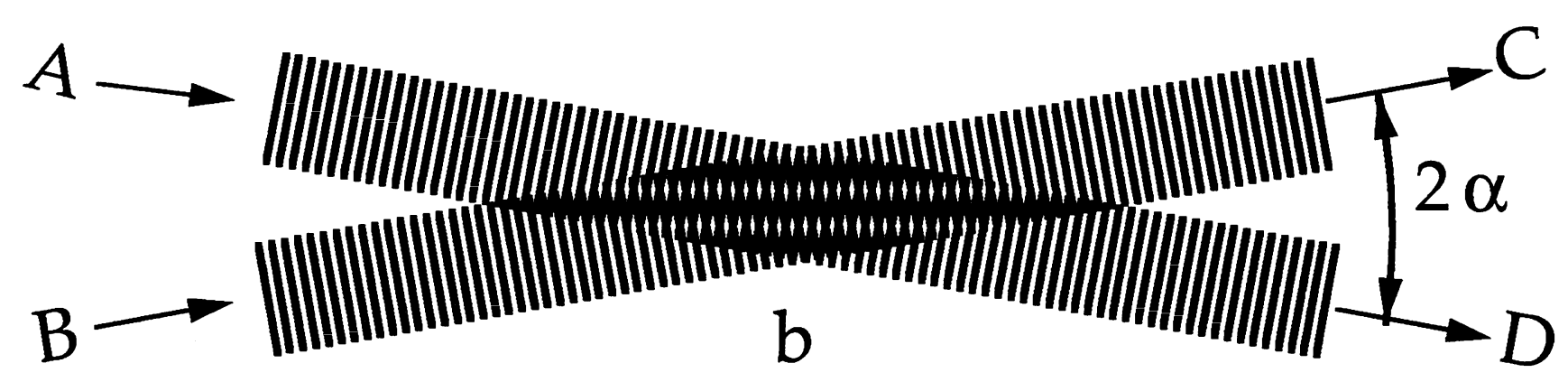

Fig. 1

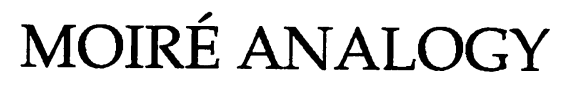

a: A transparent tape is printed with black and parallel stripes of uniform separation.

b: Two copies of (a) are placed on top of each other. Where they cross moiré fringes are formed, that bisect the angle between the tapes and are more closely spaced the larger this angle is.

\section{INTERFERENCE}

a: A coherent collimated laser beam with its light waves that are parallel and of uniform separation.

b: Two beams like (a) cross each other and moiré fringes are formed that bisect the angle between the beams and are more closely spaced the larger this angle is. 


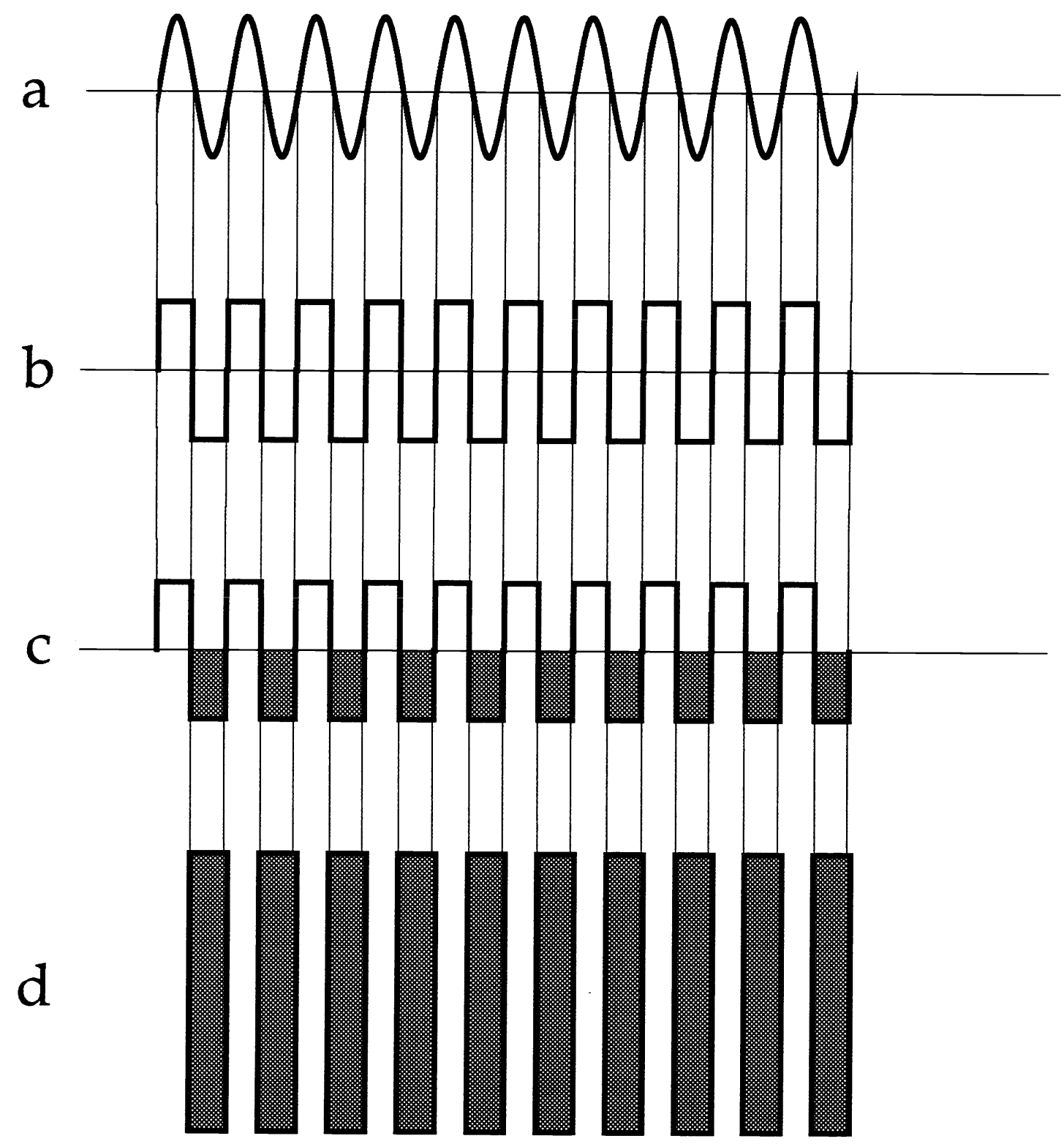

Fig. 2

a: The lightwaves of the laserbeam are represented by a sine wave.

b: The sine wave is approximated by a square curve.

C: The negative parts of the curve are painted dark.

d: The resulting striped tape used for the moiré analogies of this book. 


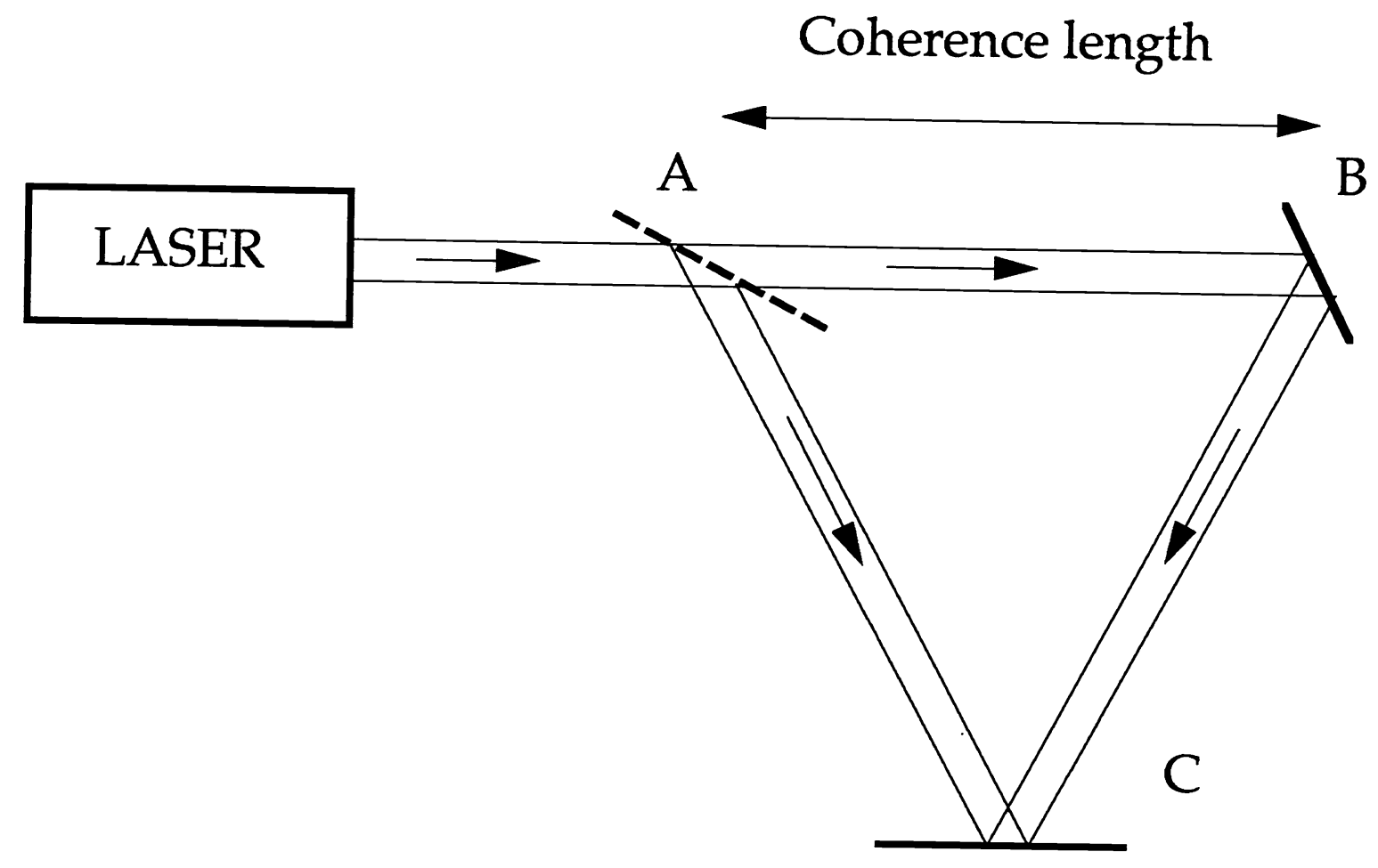

Fig. 3

The laser beam passes through a beamsplitter (A) and half of it is reflected by the mirror (B) towards a screen (C). The other half is reflected by the beam splitter to the same spot on the screen. Dark interference fringes are seen where the two beams intersect only if the distance between (A) and (B) is shorter than the coherence length of the laser light. 

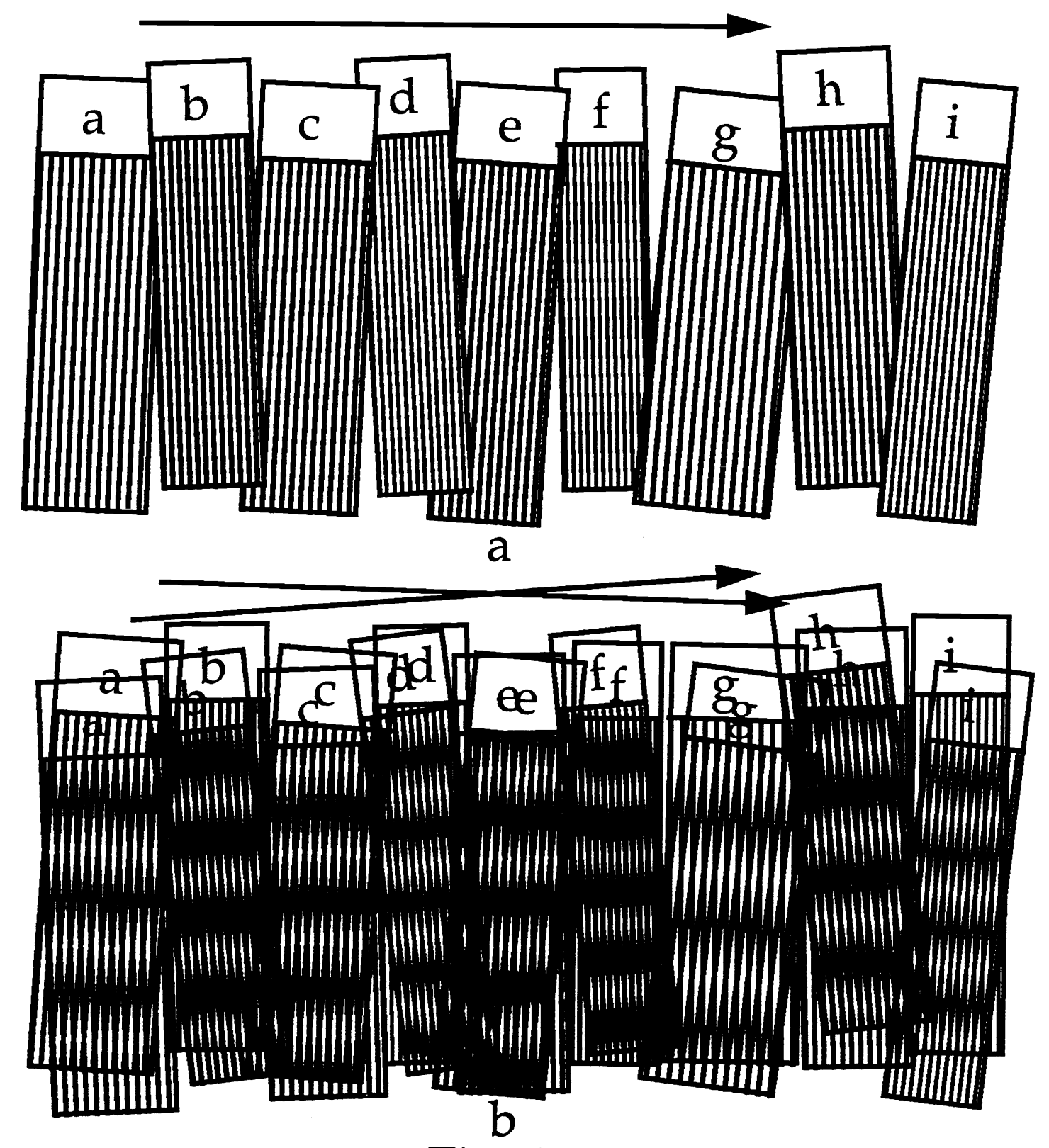

Fig. 4

MOIRÉ ANALOGY

a: Sections of stripes that are neither equidistant or parallel.

b: Even when identical sections coincide, the two copies of (a) produce moiré fringes that are neither equidistant or parallel.

INTERFERENCE

a: A spatially and temporally incoherent beam of white light.

b: Even when the difference in pathlength is zero the two crossing light beams produce interference fringes that are neither equidistant or parallel. Even now no fringes can be seen because the pattern moves with the speed of light to the right. 

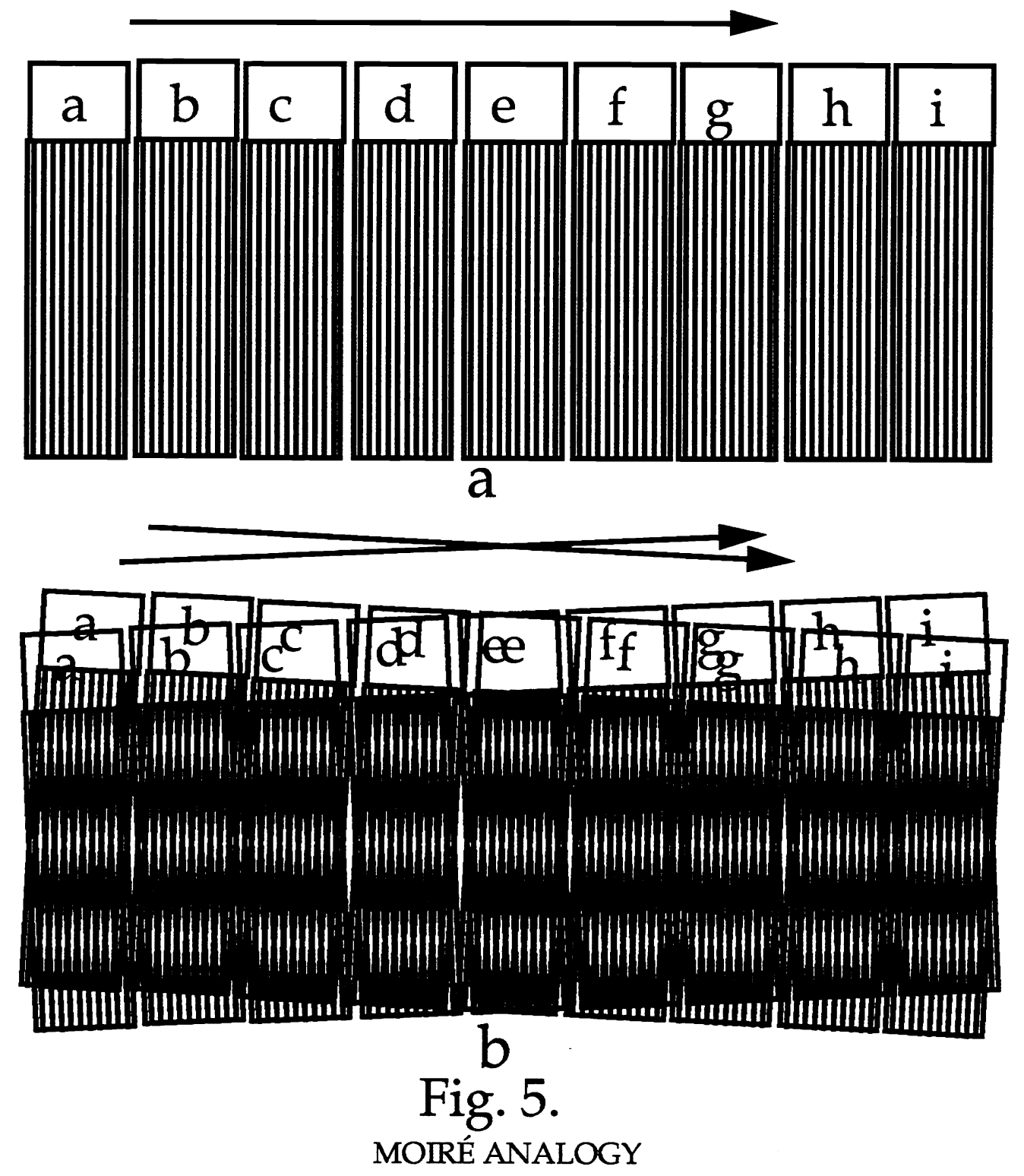

a: Sections of stripes that are equidistant and parallel, but the separation of the sections varies in a random way

b: When identical sections coincide thetwo copies of (a) produce moiré fringes that are equidistant and parallel

INTERFERENCE

a: A beam of laser light with good spatial but limited temporal coherence.

b: When the difference in path length is zero the two crossing laser beams produce interference fringes that are equidistant and parallel. Good stationary fringes can be seen when the pattern moves with the speed of light to the right. 


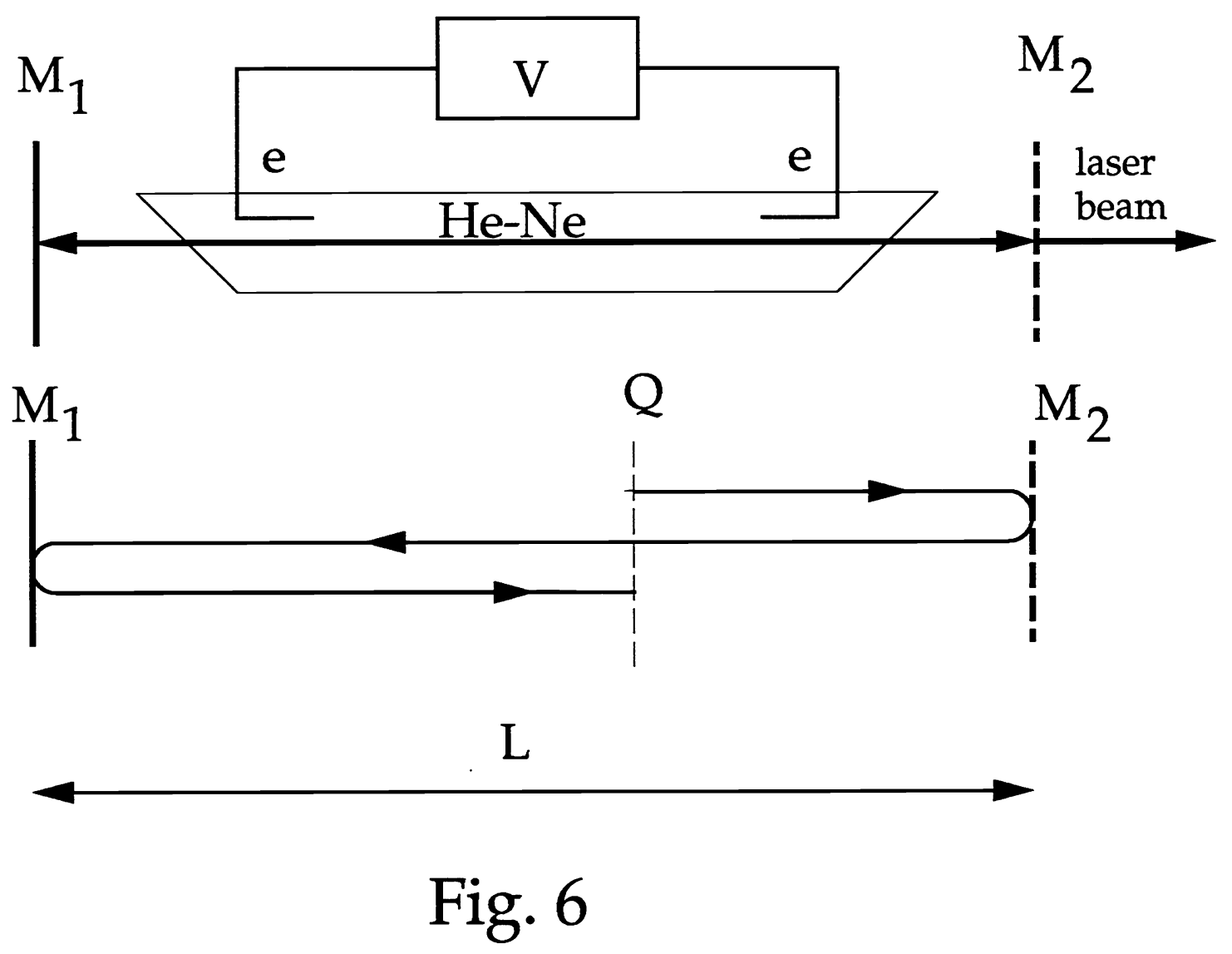

A He-Ne-laser consists of a tube with the two gases, helium and neon, at a low pressure. Two electrodes (e) with a voltage difference around 1000 volt make the gasses luminous as in a fluorescent tube. The difference is, however, that the laser usually has at each end an inclined window and a mirror that is perpendicular to the tube. Light that is reflected back and forth by the mirrors is amplified each time it passes through the gas. Each time light reaches the mirror $\mathrm{M}_{2}$, which is slightly transparent, some of it passes through and produces the laserbeam. If the light has a limited coherence length, the same coherent part will be found again and again along the beam separated by $2 \mathrm{~L}$.

Let us examine a special point in the tube ( e.g. Q). The phase of the light at $Q$ should be the same as before when it after two reflctions arrives back to $Q$, if it is out of phase there will be destructive interference and the light disappears. Thus, the laserlight will only have those wave lengths that make up an integral part of the length $2 \mathrm{~L}$. 


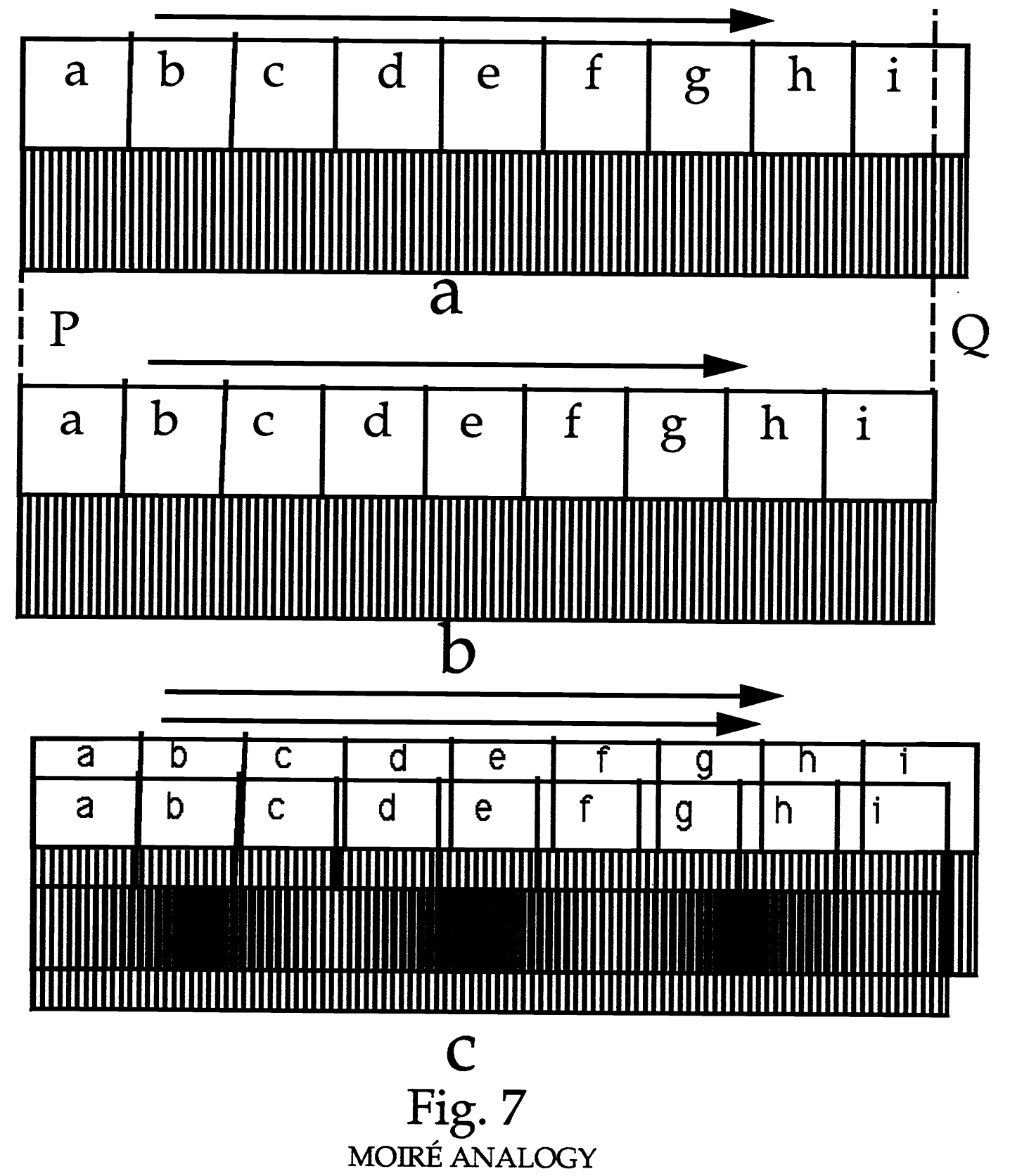

a: Parallel stripes of uniform separation, covering all sections.

b: Same as (a) but compressed so that there are 3 stripes more from (P) to $(\mathrm{Q})$.

C: (a) plus (b) produce a moiré pattern with 3 fringes.

INTEREFERENCE

a: A collimated, monochromatic and coherent laser beam.

b: Same as (a) but with a slightly shorter wavelength.

c: The two laser beams produce a beat frequency as the pattern moves with the speed of light to the right. 